\title{
Evaluación de la composición y calidad del color de vinos tintos Tannat elaborados por maceración pre-fermentativa en caliente
}

\section{Evaluation of the composition and color quality of Tannat red wines produced by hot pre-fermentative maceration}

\author{
D. Piccardo ${ }^{1, a}$, G. Favre ${ }^{1}$, O. Pascual ${ }^{2}$, J.M. Canals², F. Zamora², y G. González-Neves ${ }^{1}$ \\ ${ }^{1}$ Unidad de Tecnología de los Alimentos, Facultad de Agronomía. UDELAR. Garzón 780, Montevideo, Uruguay \\ ${ }^{2}$ Departament de Bioquímica i Biotecnologia, Facultat d'Enologia de Tarragona, Grup de Recerca en Tecnologia Enològica \\ (TECNENOL), Universitat Rovira i Virgili, Campus de Sescelades, C/Marcel.li Domingo s/n, 43007 Tarragona, Spain
}

\begin{abstract}
Resumen. Durante la vendimia 2016 se elaboraron vinos tintos Tannat por maceración pre-fermentativa en caliente (una hora de calentamiento a $60-70{ }^{\circ} \mathrm{C}$ seguido de una maceración fermentativa de 7 días) y maceración tradicional (maceración fermentativa de 7 días) con el objetivo de evaluar su concentración en compuestos fenólicos y color durante la conservación. Las vinificaciones se realizaron por triplicado en recipientes de 10 litros de capacidad. Se determinó la composición básica del vino, color, concentración de fenoles totales, antocianos, catequinas, proantocianidinas y los índices de ionización, copigmentados y PVPP. Los vinos con maceración pre-fermentativa en caliente presentaron mayor concentración de antocianos y taninos, mayor porcentaje de antocianos ionizados, co-pigmentados y condensados. Adicionalmente, presentaron mayor proporcion de delfinidina, petunidina y peonidina al descube. El calentamiento degrada las enzimas polifenoloxidasas por lo que estas formas antociánicas pudieron haber sido preservadas de la oxidación. Las concentraciones de las diferentes formas antociánicas disminuyeron durante la conservación de los vinos de ambos tratamientos, sin observarse diferencias en el perfil antocianico después de dos años. Los vinos elaborados por maceración pre-fermentativa en caliente presentaron mayor intensidad colorante durante todo el período de evaluación sugiriendo una mayor estabilidad del color.
\end{abstract}

\begin{abstract}
During the 2016 vintage, Tannat red wines were elaborated by pre-fermentative hot maceration (one hour of heating at $60-70{ }^{\circ} \mathrm{C}$ followed by a fermentative maceration of 7 days) and traditional maceration (fermentative maceration of 7 days) with the aim of evaluate its concentration in phenolic compounds and color during conservation. The vinifications were made by triplicate in containers of 10 liters of capacity. The basic wine composition, color, concentration of total phenols, anthocyanins, catechins, proanthocyanidins and the ionization, copigmented and PVPP indices were determined. The wines with pre-fermentative hot maceration showed higher concentration of anthocyanins and tannins, higher percentage of ionized anthocyanins, co-pigmented and condensed. Additionally, they presented a greater proportion of delphinidin, petunidin and peonidin at devatting. The heating degrades the polyphenoloxidases enzymes reason why these anthocyanin forms could have been preserved of the oxidation. The concentrations of the different anthocyanin forms decreased during the conservation of the wines of both treatments, without observing differences in the anthocyanin profile after two years. The wines elaborated by pre-fermentative hot maceration showed greater color intensity during the entire evaluation period, suggesting a greater color stability.
\end{abstract}

\section{Introducción}

Tannat es la variedad más implantada en Uruguay debido a su adaptación a las condiciones eco-fisiológicas del país y a la tipicidad de sus vinos. Las uvas de este cultivar se caracterizan por presentar una elevada riqueza fenólica y antocianica y una baja extractibilidad de los antocianos (altos valores de EA\%) [1]. Sus vinos presentan un perfil antociánico característico, con menores proporciones de malvidina y glucósidos acetilados, respecto a los vinos elaborados a partir de los cultivares Cabernet-Sauvignon y Merlot. En consecuencia, la estabilidad del color de los

a e-mail: dpiccardo@fagro.edu.uy vinos Tannat elaborados por maceraciones tradicionales es menor a la de los vinos de otras variedades [1-3]. Esta dificultad relativa a la extracción y composición de los antocianos pueden ser compensadas con operaciones que promuevan su solubilización y estabilización desde el inicio de la maceración a fin de aprovechar el potencial enológico de las uvas y producir vinos con mejor color y más estables en el tiempo $[1,4,5]$.

Numerosos estudios se han centrado en la extracción y la estabilización química del color. Algunas técnicas propuestas buscan romper las barreras celulares e incrementar la extracción de los antocianos favoreciendo sus interacciones con otros constituyentes del vino [5,6]. 
Otras, proponen el uso de agregados externos (taninos o manoproteinas entre otros) para estabilizar el color [7,8].

En este sentido, la maceración pre-fermentativa en caliente potencia la extracción de los compuestos fenólicos con el objetivo de obtener vinos tintos con mayor color. En el calentamiento pre-fermentativo de los racimos enteros o estrujados $[9,10]$ se alteran los tejidos de los hollejos y se transfieren sus componentes (principalmente antocianos y taninos) al mosto. Las temperaturas a las que se calienta el mosto varían entre 40 y $80^{\circ} \mathrm{C}$ y su duración entre 1 y $24 \mathrm{~h}[5,11]$. Después del tratamiento térmico, los mostos pueden continuar o no con una maceración fermentativa dependiendo del objetivo buscado. En consecuencia, la extracción de los antocianos incrementa la intensidad colorante del mosto desde el inicio de la maceración [12] favoreciendo su reacción tanto con compuestos procedentes de la fermentación alcohólica como con otros compuestos fenólicos. Adicionalmente, la copigmentación se ve favorecida mejorando la calidad del color del vino a través de un incremento en el espectro visible (efecto hipercromico) y un incremento en el máximo de absorbancia (efecto batocrómico) [13]. Sin embargo, la copigmentación disminuye si el aumento de la temperatura es lento ya que estos pigmentos son exotérmicos y su equilibrio tiende a desplazarse hacia la forma chalcona [14]. Al terminar la fermentación alcohólica los vinos resultan con más color que los elaborados por sistemas tradicionales y estando dotados de una mayor estabilidad $[9,11]$.

Esta investigación tiene como efectivo evaluar el efecto de la maceración pre-fermentativa en caliente sobre la composición fenólica y el color de vinos tintos Tannat al descube y durante su conservación.

\section{Materiales y métodos}

\subsection{Cosecha y vinificaciones}

Los vinos se elaboraron durante la vendimia 2016 con uvas del cultivar Tannat (Vitis vinifera L., Vitis International Variety Catalogue number: VIVC 12257) [15] cosechadas de un viñedo comercial ubicado en Canelón Chico, Canelones, Uruguay. La cosecha se realizó cuando las uvas alcanzaron la madurez tecnológica (Tabla 1).

Una vez cosechadas, las uvas fueron estrujadas, despalilladas y distribuidas al azar entre las distintas unidades experimentales.

Las vinificaciones se realizaron en recipientes de 10 litros de capacidad donde se encubaron $8 \mathrm{~kg}$ de uva. Tres recipientes fueron vinificados de manera tradicional (7 días de maceración fermentativa, VT) mientras que los otros tres fueron sometidos a una maceración prefermentativa en caliente $\left(60-70^{\circ} \mathrm{C}\right)$ durante una hora, seguida de una maceración fermentativa de 7 días (MPC). El calentamiento se llevó a cabo transfiriendo el mosto, hollejos y semillas a recipientes de acero inoxidable de $11 \mathrm{~L}$ de capacidad que se sumergieron en un baño de agua caliente $\left(80-90^{\circ} \mathrm{C}\right)$. Durante el calentamiento, el mosto se homogeneizó manualmente. Al final del tratamiento térmico, los recipientes de acero inoxidable se sumergieron en un baño de agua fría para refrigerarlos a temperatura ambiente (alrededor de $26^{\circ}$ C). Posteriormente, el mosto, hollejos y semillas se transfirieron a los recipientes originales donde se continuó con una maceración fermentativa de 7 días.
Todos los tanques se inocularon con $200 \mathrm{mg} / \mathrm{L}$ de levadura seca activa (Saccharomyces cerevisiae ex bayanus Natuferm 804; Oenobiotech, Paris, Francia). Durante la maceración, se realizó un remontaje diario a fin de favorecer la extracción de polifenoles. La temperatura de fermentación estuvo comprendida entre 25 y $29^{\circ} \mathrm{C}$. Después de 7 días de maceración, el vino se decantó por gravedad y los orujos se prensaron ligeramente en una prensa manual. El vino se mantuvo en recipientes de $5 \mathrm{~L}$ de capacidad a temperatura ambiente. Una vez finalizada la fermentación maloláctica espontánea, todos los vinos se estabilizaron con $100 \mathrm{mg} / \mathrm{l}$ de $\mathrm{K}_{2} \mathrm{~S}_{2} \mathrm{O}_{2}$ y $300 \mathrm{mg} / \mathrm{l}$ de lisozima (Delvo ${ }^{\circledR}$ Zyme, Delft, Países Bajos). Finalmente, los vinos fueron embotellados y almacenados hasta el análisis. Los análisis se realizaron a los 2 meses del descube y se repitieron a los 1 y 2 años.

\subsection{Análisis de la composición general de las uvas y los vinos elaborados}

Los métodos analíticos recomendados por la Organización Internacional de la Viña y el Vino [16] se utilizaron para determinar la concentración de azúcares, el pH y la acidez titulable de los mostos. El contenido total de antocianinas de las uvas, su extractibilidad y riqueza fenólica se determinó de acuerdo con el método de Glories y Augustien [17] modificado por González-Neves et al. [1].

El contenido de etanol, la acidez titulable y el pH de los vinos se determinaron a los dos meses del descube utilizando el analizador de infrarrojos Winescan TM Autosampler 79000 (Foss, EE. UU.) y el software Foss Integrator versión 154 (Foss, Dinamarca).

\subsection{Determinación de los parámetros cromáticos}

Los parámetros de color se determinaron directamente en las muestras de vino colocadas en una cubeta de $1 \mathrm{~mm}$ de recorrido óptico. La intensidad colorante (IC) se estimó utilizando el método descrito por Glories [18]. Las coordenadas CIELAB, luminosidad ( $\mathrm{L}^{*}$ ), cromaticidad $\left(\mathrm{C}^{*}\right)$ y tono $\left(\mathrm{H}^{*}\right)$, se determinaron de acuerdo con el método de Ayala et al. [19] y el procesamiento de datos se realizó utilizando el software MSCV [20].

\subsection{Análisis espectrofotométricos de la composición fenólica de los vinos y parámetros relacionados}

La composición polifenólica se evaluó utilizando índices espectrofotométricos clásicos. Los polifenoles totales se determinaron usando el Folin-Ciocalteu, de acuerdo con Singleton y Rossi [21], y sus contenidos en los vinos se expresan en $\mathrm{mg}$ de ácido gálico por litro. La concentración de antocianos fue analizada por RibéreauGayon y Stonestreet [22] y se expresan en mg de malvidin3-glucósido equivalente (EMG) por litro. Las catequinas se cuantificaron usando el método de Swain y Hillis [23] y sus concentraciones se expresan en mg de D-catequina por litro. Las proantocianidinas se determinaron según Ribéreau-Gayon y Stonestreet [22] y sus contenidos se expresan en $\mathrm{mg}$ de cloruro de cianidina por litro de vino. El índice de ionización (que indica la proporción de antocianos que presentan color rojo al $\mathrm{pH}$ del vino) y el índice de PVPP (que indica la proporción de antocianinas 
Tabla 1. Composición inicial de la uva.

\begin{tabular}{cccccc}
\hline $\begin{array}{c}\text { Azúcares } \\
(\mathbf{m g} / \mathbf{L})\end{array}$ & $\begin{array}{c}\text { Acidez titulable } \\
\left(\mathbf{m g ~ H}_{2} \mathbf{S O}_{4} / \mathbf{L}\right)\end{array}$ & $\mathbf{p H}$ & $\mathbf{A 2 8 0}$ & $\begin{array}{c}\mathbf{A p H 1} \\
(\mathbf{m g} / \mathbf{L})\end{array}$ & EA (\%) \\
\hline $243 \pm 3$ & $4.51 \pm 0.07$ & $3.31 \pm 0.03$ & $47.5 \pm 0.5$ & $2258 \pm 145$ & $51.6 \pm 2.6$ \\
\hline
\end{tabular}

A280: riqueza fenlica de la uva; ApH1: potencial en antocianos totales, EA: extractibilidad de los antocianos.

Tabla 2. Composición básica de los vinos.

\begin{tabular}{cccc}
\hline & $\begin{array}{c}\text { Alcohol } \\
(\% \mathbf{v} / \mathbf{v})\end{array}$ & $\begin{array}{c}\text { Acidez titulable } \\
\left(\mathbf{g} / \mathbf{L H}_{2} \mathbf{S O}_{4}\right)\end{array}$ & $\mathbf{p H}$ \\
\hline VT & $14.6 \pm 0.2 \mathrm{a}$ & $3.88 \pm 0.19 \mathrm{a}$ & $4.01 \pm 0.13 \mathrm{a}$ \\
MPC & $14.8 \pm 0.1 \mathrm{a}$ & $3.76 \pm 0.03 \mathrm{a}$ & $4.09 \pm 0.01 \mathrm{a}$ \\
\hline
\end{tabular}

Medias con distinta letra indican diferencias significativas $(p<0.05)$ VT: vinificación tradicional; MPC: maceración pre-fermentativa en caliente.

combinadas con proantocianidinas) se determinaron de acuerdo con Glories [18]. El índice de copigmentación se midió de acuerdo con Boulton [24].

\subsection{Determinación de antocianos por HPLC}

Los análisis de HPLC en fase reversa de las antocianinas se llevaron a cabo inyectando $40 \mu \mathrm{L}$ de vino en un cromatógrafo líquido Agilent serie 1200 (HPLCDAD) y utilizando una columna Agilent Zorbax Eclipse XDBC18, $4.6 \times 250 \mathrm{~mm}, 5 \mu \mathrm{m}$ (Agilent Technologies). Los disolventes utilizados fueron ácido fórmico acuoso al $10 \%$ (disolvente A) y una mezcla de metanol al $45 \%$, agua al $45 \%$ y ácido fórmico al $10 \%$ (disolvente B) de acuerdo con el método descrito por Valls [25]. Los cromatogramas se registraron a $520 \mathrm{~nm}$, y las curvas estándar de antocianina se realizaron usando malvidin-3-O-glucósido cloruro. Los compuestos se identificaron registrando sus espectros UV con el detector de matriz de diodos y comparándolos con los espectros UV reportados en la literatura. Se cuantificaron los cinco antocianidin-3-monoglucósidos del vino (delfinidina, cianidina, peonidina, petunidina $\mathrm{y}$ malvidina) y sus respectivas antocianinas acetiladas y p-cumarilados.

\subsection{Análisis estadistico}

Todos los datos se expresan como el promedio aritmético \pm desviación estándar de tres repeticiones. Las diferencias estadísticas entre los tratamientos fueron determinadas aplicando análisis de varianza. Los análisis estadísticos se realizaron utilizando el software Infostat versión 2015 [26].

\section{Resultados y discusión}

\subsection{Composición básica de los vinos}

La Tabla 2 muestra el contenido de etanol, la acidez titulable y el pH de los vinos Tannat elaborados por vinificación tradicional (VT) y maceración prefermentativa en caliente (MPC).

Los vinos MPC no mostraron diferencias significativas en su composición básica respecto a los vinos VT. Varias investigaciones han reportado un incremento en el contenido de alcohol y una disminución del $\mathrm{pH}$ en los vinos elaborados por maceración pre-fermentativa en caliente [27]. Este efecto no se observó en nuestra investigación.

\subsection{Composición fenólica de los vinos y parámetros relacionados}

La Tabla 3 muestra la composición fenólica de los vinos VT y MPC a los dos meses del descube (0), 1 y 2 años.

Las concentraciones de polifenoles totales, antocianos, catequinas y proantocianidinas de los vinos MPC a los dos meses del descube y durante su conservación fueron significativamente mayores respecto a las de los vinos VT. Estos resultados concuerdan con los reportados por otros autores quienes observaron una mayor de los compuestos fenólicos en los vinos que fueron sometidos a un tratamiento térmico pre-fermentativo del mosto $[9,11,27]$. Esta investigación demuestra que las diferencias en la composición fenólica de los vinos se mantienen hasta los dos años posteriores a la primera determinación analítica.

Durante el primer año de conservación, la concentración de polifenoles totales de los vinos VT y MPC disminuye significativamente, estabilizándose hacia el segundo año. Por su parte, la concentración de antocianos de los vinos de ambos tratamientos disminuyó significativamente durante todo el período de evaluación. Estos compuestos experimentan fenómenos de precipitación que pueden ocurrir tanto en los vinos jóvenes como en los envejecidos. En los vinos jóvenes se relaciona con el estado coloidal de la materia colorante y con el tamaño molecular, y depende del contenido alcohólico del vino y de la temperatura de la conservación [28]. En los vinos envejecidos está más relacionado con las reacciones de estos pigmentos con otros constituyentes del vino. La copigmentación [29]; la formación de piranoantocianos [30], la reacción directas tanino-antocianos [31] o la reacción tanino-antociano mediada por el acetaldehído [32] pueden estabilizar a los antocianos, determinando una disminución en su concentración original y un cambio en el color del vino.

La concentración de catequinas aumento significativamente durante la conservación de los vinos de ambos tratamientos en cambio las proantocianidinas disminuyeron, aunque la disminución en la concentracion de éstas últimas no fue significativa. Los taninos sufren reacciones de ruptura durante la conservación que generan moléculas de bajo peso molecular [30,33], lo cual podría estar explicando el incremento en la concentración de catequinas observado.

La Tabla 4 muestra el índice de ionización, copigmentación y PVPP determinados a los dos meses del descube para los vinos de VT y MPC. Los vinos de MPC presentaron índices más altos de ionización, copigmentación y PVPP. Estos resultados son consistentes con los obtenidos en la composición fenólica de los vinos (Tabla 3) y reafirman la hipótesis de que la maceración 
Tabla 3. Composición fenólica de los vinos.

\begin{tabular}{|c|c|c|c|c|c|}
\hline & & $\begin{array}{c}\text { Polifenoles } \\
\text { totales }(\mathrm{mg} / \mathrm{L})\end{array}$ & $\begin{array}{l}\text { Antocianos } \\
(\mathrm{mg} / \mathrm{L})\end{array}$ & $\begin{array}{l}\text { Catequinas } \\
(\mathrm{mg} / \mathrm{L})\end{array}$ & $\begin{array}{c}\text { Proantocianidinas } \\
(\mathrm{mg} / \mathrm{L})\end{array}$ \\
\hline \multirow{3}{*}{ VT } & $\mathbf{0}$ & $2377 \pm 127$ a,B & $1031 \pm 52 \mathrm{a}, \mathrm{B}$ & $1189 \pm 101 \mathrm{c}, \mathrm{B}$ & $3711 \pm 107 \mathrm{a}, \mathrm{B}$ \\
\hline & $1^{\text {er }}$ año & $2084 \pm 123$ b,B & $566 \pm 33 \mathrm{~b}, \mathrm{~B}$ & $1408 \pm 103 \mathrm{~b}, \mathrm{~B}$ & $3472 \pm 199 \mathrm{a}, \mathrm{B}$ \\
\hline & $2^{\text {do }}$ año & $2007 \pm 64 \mathrm{~b}, \mathrm{~B}$ & $348 \pm 16 \mathrm{c}, \mathrm{B}$ & $1741 \pm 28 \mathrm{a}, \mathrm{B}$ & $3345 \pm 139$ a,B \\
\hline \multirow{3}{*}{ MPC } & $\mathbf{0}$ & $2639 \pm 126 \mathrm{a}, \mathrm{A}$ & $1260 \pm 148 \mathrm{a}, \mathrm{A}$ & $1862 \pm 141 \mathrm{c}, \mathrm{B}$ & $4677 \pm 113 \mathrm{a}, \mathrm{A}$ \\
\hline & $1^{\text {er }}$ año & $2554 \pm 165 \mathrm{~b}, \mathrm{~A}$ & $712 \pm 79 \mathrm{~b}, \mathrm{~A}$ & $2125 \pm 123 \mathrm{~b}, \mathrm{~A}$ & $4564 \pm 165 \mathrm{a}, \mathrm{A}$ \\
\hline & $2^{\text {do }}$ año & $2486 \pm 149 \mathrm{~b}, \mathrm{~A}$ & $438 \pm 15 \mathrm{c}, \mathrm{A}$ & $2454 \pm 139$ a,A & $4435 \pm 112 \mathrm{a}, \mathrm{A}$ \\
\hline
\end{tabular}

Letras minúsculas indican diferencias significativas entre los momentos de análisis para un mismo tratamiento $(p<0.05)$. Letras mayúsculas indican diferencias significativas entre los tratamientos para un momento de análisis $(p<0.05)$. VT: vinificación tradicional; MPC: maceración pre-fermentativa en caliente.

Tabla 4. Composición fenólica de los vinos.

\begin{tabular}{lccc}
\hline & $\begin{array}{c}\text { Indice de } \\
\text { Ionización (\%) }\end{array}$ & $\begin{array}{c}\text { Índice de } \\
\text { Copigmentación }(\%)\end{array}$ & $\begin{array}{c}\text { Índice de PVPP } \\
(\%)\end{array}$ \\
\hline VT & $25.6 \pm 2.8 \mathrm{~b}$ & $12.2 \pm 2.0 \mathrm{~b}$ & $39.4 \pm 4.7 \mathrm{~b}$ \\
MPC & $42.2 \pm 6.2 \mathrm{a}$ & $20.7 \pm 0.7 \mathrm{a}$ & $51.0 \pm 8.8 \mathrm{a}$ \\
\hline
\end{tabular}

Medias con distinta letra indican diferencias significativas $(p<0.05)$ VT: vinificación tradicional; MPC: maceración pre-fermentativa en caliente.
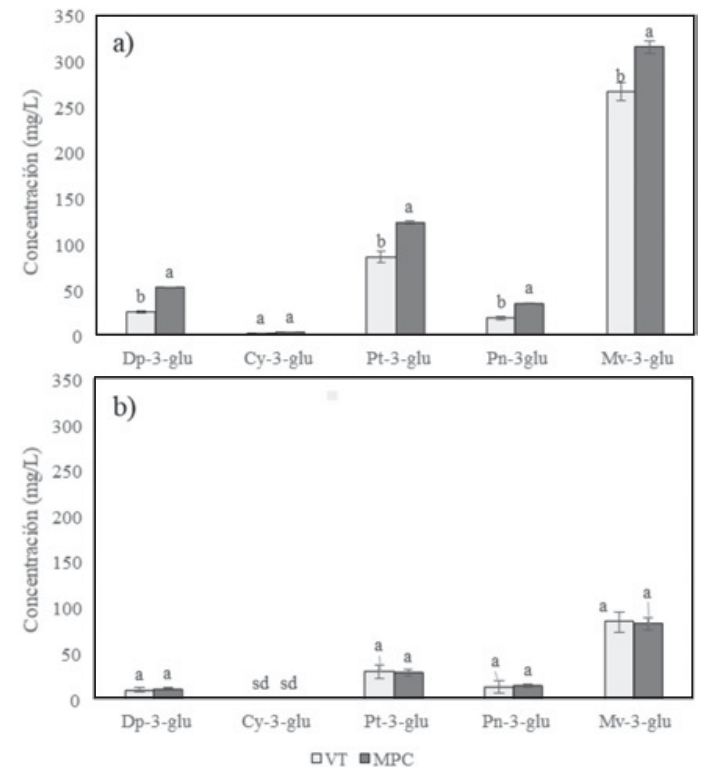

Figura 1. Concentración de antocianos a dos meses del descube (a) y a los dos años (b) Medias con distinta letra indican diferencias significativas $(p<0.05)$. VT: vinificación tradicional; MPC: maceración pre-fermentativa en caliente; Dp-3-glu: Delfinidina-3-glucósido; Cy-3-glu: Cianidina-3-glucósido; Pt-3glu: Petunidina-3-glucósido; Pn-3-glu: Peonidina-3-glucósido; Mv-3-glu: Malvidina-3-glucósido.

pre-fermentativa en caliente favorece las interacciones entre los antocianos y otros constituyentes del vino promoviendo la copigmentación y la formación de aductos entre antocianos y taninos.

\subsection{Composición antociánica de los vinos y parámetros relacionados}

La concentración de las diferentes formas antociánicas de los vinos VT y MPC a dos meses del descube y a los dos años se observa en la Fig. 1a y b respectivamente.

A los dos meses del descube, los vinos MPC presentaron mayor concentración de todas las formas antociánicas respecto a los vinos VT, con excepción de la cianidina-3-O-glucósido donde no se encontraron
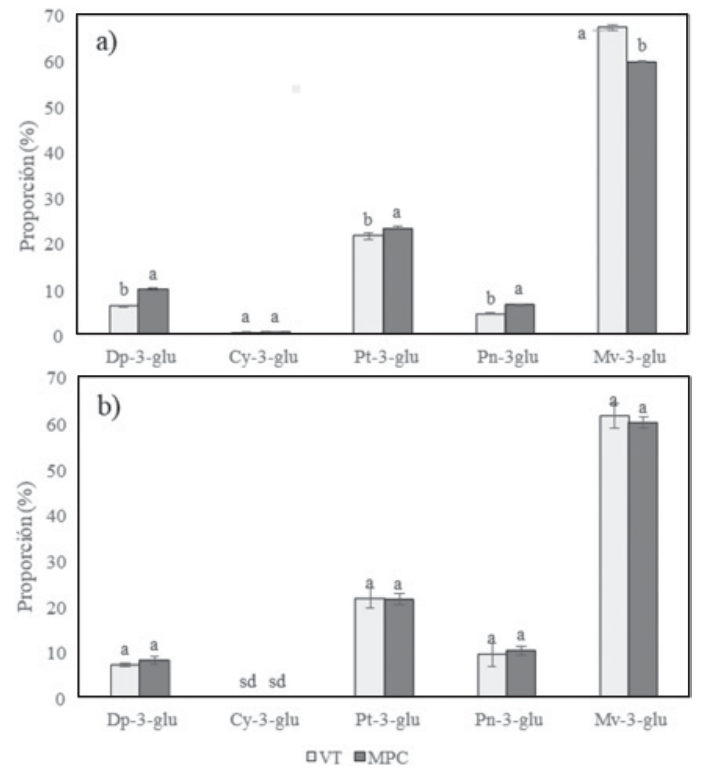

Figura 2. Proporción de antocianos a dos meses del descube (a) y a los dos años (b) Medias con distinta letra indican diferencias significativas $(p<0.05)$. VT: vinificación tradicional; MPC: maceración pre-fermentativa en caliente; Dp-3-glu: Delfinidina-3-glucósido; Cy-3-glu: Cianidina-3-glucósido; Pt-3glu: Petunidina-3-glucósido; Pn-3-glu: Peonidina-3-glucósido; Mv-3-glu: Malvidina-3-glucósido.

diferencias significativas (Fig. 1a). Estos resultados concuerdan con las concentraciones totales de antocianos obtenidas por espectrofotometría y son explicados por una mayor extracción de estos durante el tratamiento térmico pre-fermentativo. Para los vinos de ambos tratamientos, la concentración de todas las formas antociánicas disminuyó a los dos años respecto a su concentración original (Fig. 1b). En este sentido, la ausencia de diferencias significativas entre los tratamientos sugiere que los vinos MPC tuvieron una mayor disminución en la concentración de estos pigmentos lo cual podría estar relacionado con una mayor formación de pigmentos derivados.

La Fig. 2a y b muestra los perfiles antociánicos de los vinos VT y MPC a los dos meses del descube y a los dos años respectivamente.

Los perfiles antociánicos de los vinos VT y MPC fueron significativamente diferentes a los dos meses del descube (Fig. 2a). Los vinos MPC presentaron mayor porcentaje de delfinidina, petunidina y peonidina y menor de malvidina respecto a los vinos VT. La malvidina es más resistente a la degradación térmica que otras 
Tabla 5. Color de los vinos.

\begin{tabular}{cccccc}
\hline & & $\begin{array}{c}\text { Intensidad } \\
\text { colorante }\end{array}$ & $\mathbf{L}^{*}$ & $\mathbf{C}^{*}$ & $\mathbf{H}^{*}$ \\
\hline \multirow{2}{*}{$\mathbf{V T}$} & $\mathbf{0}$ & $26.7 \pm 0.9 \mathrm{a}, \mathrm{B}$ & $38.6 \pm 1.3 \mathrm{a}, \mathrm{A}$ & $42.4 \pm 0.5 \mathrm{~b}, \mathrm{~A}$ & $346.5 \pm 1.0 \mathrm{a}, \mathrm{A}$ \\
& $\mathbf{1}^{\text {er }}$ año & $18.4 \pm 0.4 \mathrm{~b}, \mathrm{~B}$ & $33.3 \pm 2.2 \mathrm{~b}, \mathrm{~A}$ & $48.9 \pm 0.4 \mathrm{a}, \mathrm{A}$ & $13.6 \pm 1.3 \mathrm{c}, \mathrm{A}$ \\
& $\mathbf{2}^{\text {do }}$ año & $17.9 \pm 0.1 \mathrm{~b}, \mathrm{~B}$ & $34.3 \pm 1.7 \mathrm{~b}, \mathrm{~A}$ & $48.5 \pm 0.1 \mathrm{a}, \mathrm{A}$ & $23.4 \pm 1.5 \mathrm{~b}, \mathrm{~A}$ \\
& $\mathbf{0}$ & $34.7 \pm 1.5 \mathrm{a}, \mathrm{A}$ & $27.6 \pm 1.6 \mathrm{c}, \mathrm{B}$ & $43.8 \pm 2.2 \mathrm{~b}, \mathrm{~A}$ & $348.1 \pm 0.8 \mathrm{a}, \mathrm{A}$ \\
MPC & $\mathbf{1}^{\text {er }}$ año & $27.2 \pm 1.1 \mathrm{~b}, \mathrm{~A}$ & $20.4 \pm 1.7 \mathrm{~b}, \mathrm{~B}$ & $49.7 \pm 0.4 \mathrm{a}, \mathrm{A}$ & $9.1 \pm 3.8 \mathrm{c}, \mathrm{A}$ \\
& $\mathbf{2}^{\text {do }}$ año & $23.0 \pm 0.4 \mathrm{c}, \mathrm{A}$ & $22.6 \pm 0.8 \mathrm{~b}, \mathrm{~B}$ & $47.7 \pm 1.3 \mathrm{a}, \mathrm{A}$ & $21.3 \pm 2.3 \mathrm{~b}, \mathrm{~A}$ \\
\hline
\end{tabular}

Letras minúsculas indican diferencias significativas entre los momentos de análisis para un mismo tratamiento $(p<$ 0.05). Letras mayúsculas indican diferencias significativas entre los tratamientos para un momento de análisis $(p<0.05)$. VT: vinificación tradicional; MPC: maceración pre-fermentativa en caliente.

antocianidinas [11], por lo que no debería ser degradada durante el tratamiento térmico. Por otra parte, se ha demostrado que el calentamiento pre-fermentativo por encima de $60{ }^{\circ} \mathrm{C}$ degrada las enzimas polifenoloxidasas, responsables de la oxidación de los compuestos fenólicos en las primeras etapas de la vinificación [12,28]. Debido a que los hidroxilos adyacentes de los grupos O-difenol son sensibles a la oxidación, el malvidin-3-O-glucósido y peonidin-3-O-glucósido que no poseen grupos hidroxilo en posición orto son comparativamente más resistentes a la oxidación que la cianidina-3-O-glucósido [34]. Podría pensarse que el aumento en las proporciones de delfinidina, petunidina y peonidina se debe al hecho de que estas formas fueron preservadas de la oxidación enzimática durante la vinificación.

A los dos años no se observaron diferencias en los perfiles antociánicos de los vinos de ambos tratamientos (Fig. 2b). Es posible que delfinidina, petunidina $y$ peonidina, que se encontraban en mayor proporción en los vinos MPC al descube, hayan sido las más afectadas durante la conservación respecto a las otras formas antocíanicas. En este sentido, estos pigmentos son más susceptibles al proceso de oxidación no enzimática que ocurre durante la conservación del vino [34].

\subsection{Color}

Los parámetros de color de los vinos VT y MPC se muestran en la Tabla 5.

A los dos meses del descube, los vinos MPC presentaron un color más intenso que los VT. Específicamente, la intensidad colorante (IC) fue significativamente más alta y la luminosidad (L*) significativamente más baja. Estos resultados son explicados fundamentalmente por la mayor concentración de antocianos y mayores valores de los índices de ionización, copigmentación y PVPP de los vinos MPC. La cromaticidad $\left(\mathrm{C}^{*}\right)$ y el tono $\left(\mathrm{H}^{*}\right)$ fue mayor en los vinos MPC, pero estas diferencias no fueron significativas.

Si bien la intensidad colorante disminuyó significativamente durante la conservación de los vinos de ambos tratamientos, los vinos MPC mantuvieron los mayores valores luego de dos años de evaluación. En este sentido, el aumento en la extracción de antocianinas desde las primeras etapas de la maceración y el aumento en la extracción de taninos permite una mayor asociación de estas moléculas, lo que se ha reportado como un factor determinante para mejorar la estabilización del color [24,25]. La luminosidad de los vinos disminuyó únicamente durante el primer año de conservación, siendo los vinos MPC los más oscuros. Este resultado no resulta muy lógico ya que la disminución en la intensidad del color implicaría un incremento en la luminosidad y no una disminución como fue observado en nuestros resultados. La cromaticidad se incrementó en ambos vinos durante el primer año, sin observarse diferencias significativas entre los tratamientos. Por otra parte, los vinos pasaron de tonalidades $\left(\mathrm{H}^{*}\right)$ rojo-azuladas al descube a tonalidades más rojo-amarillentas a los dos años. Esta evolución del color es la esperada ya que la mayoría de los pigmentos derivados poseen color amarillo-naranja y contribuyen al cambio de color durante el envejecimiento del vino tinto [34].

\section{Conclusiones}

La maceración prefermentativa en caliente permitió elaborar vinos Tannat con mayor concentración de compuestos fenólicos e intensidad colorante al descube y hasta los dos años de conservación.

En particular, los vinos elaborados con esta técnica presentaron mayor concentración de compuestos fenólicos totales, antocianos, catequinas y proantocianidinas al descube, 1 y 2 años de conservación. Al descube, el perfil antocianico de los vinos fue modificado por la maceración pre-fermentativa en caliente, en donde se preservan las formas antociánicas más susceptibles a la oxidación enzimática. Sin embargo, estas formas antociánicas son degradadas durante la conservación del vino. La intensidad del color de los vinos MPC fue significativamente mayor al descube y durante la conservación, sugiriendo un color más estable. Estos resultados están de acuerdo con los perfiles antociánicos de los vinos y las mayores concentraciones de antocianos y taninos que podrían favorecer la formación de pigmentos derivados. Sin embargo, los parámetros cromáticos relacionados a la calidad del color no fueron afectados por esta técnica de vinificación.

Futuras investigaciones deben centrarse en determinar el efecto de la maceración pre-fermentativa en caliente sobre la evolución del perfil antociánico de los vinos y la formación de pigmentos poliméricos durante la conservación del vino.

Los autores agradecen al Instituto Nacional de Vitivinicultura (I.NA.VI.), Establecimiento Juanicó y Bodega Olga Silva El trabajo contó con el apoyo financiero de la CAP (Comisión Académica de Posgrado de la Universidad de la República), ANII (Agencia Nacional de Investigación e Innovación, MOV_CA_2015_1_107599) y CISC (Comisión Sectorial de Investigación Científica, beca de Movilidad, 2016 y 2017. 


\section{References}

[1] G. González-Neves, L. Barreiro, G. Gil, J. Franco, A. Carbonneau, M. Moutounet, Bull. OIV 887-888, 30 (2005)

[2] G. González-Neves, L. Barreiro, G. Gil, J. Franco, M. Ferrer, A. Carbonneau, M. Moutounet, Anal. Chim. Acta 513, 197 (2004)

[3] G. González-Neves, J. Franco, L. Barreiro, G. Gil, M. Moutounet, A. Carbonneau, Eur. Food Res. Technol. 225, 111 (2007)

[4] Y. Glories, Progres agricole et viticole 118, 347 (2001)

[5] K. Sacchi, L. Bisson, D. Adams, Am. J. Enol. Vitic. 56, 197 (2005)

[6] N. López, E. Puértolas, S. Condón, I. Alvarez, J. Raso, Eur. Food Res. Technol. 227, 1099 (2008)

[7] J. Gombau, A. Vignault, O. Pascual, J.M. Canals, P.L. Teissedre, F. Zamora, BIO Web of Conferences 7, 02033 (2016)

[8] C. Alcalde-Eón, I. García-Esteìvez, V. Puente, J.C. Rivas-Gonzalo, M.T. Escribano-Bailoìn, J. Agric. Food Chem. 62, 6984 (2014)

[9] N. El Darra, F. Turk, M. Ducasse, N. Grimi, R. Maroun, N. Louka, E. Vorobiev, Food Chem. 194, 944 (2016)

[10] R. Boulton, V. Singleton, L. Bisson, R. Kukkee, Zaragoza. Acrinia S.A. 635 (2002)

[11] M. Atanackovic, A. Petrovic, S. Jovic, L. GojkovicBukarica, Food Chem. 131, 513 (2012)

[12] N. Andrade-Neves, L. Araujo-Panto, A. Soares dos Santos, A. J. Food Eng. 97, 384 (2014)

[13] M. Abyari, R. Heidari, R. Jamei, J. Biol. Sci. 6, 638 (2006)

[14] R. Brouillard, O. Dangles, Food Chem. 51, 365 (1994)

[15] Vitis International variety Catalogue, http:// www.vivc.de/

[16] OIV. Vol. 1. Paris, France: Organisation Internationale de la Vigne et du Vin (2018)

[17] Y. Glories, M. Augustin, In: C. R. Colloque Journée Techn. (CIVB, Bordeaux, 1993), p. 56
[18] Y. Glories, Conn. Vigne Vin 18, 195 (1984)

[19] F. Ayala, J.F. Echávarri, A.I. Negueruela, Am. J. Enol. Vitic. 48, 357 (1997)

[20] F. Ayala, J.F. Echávarri, A.I. Negueruela, MSCVes.zip. URL http://www.unizar.es/ negueruela/MSCV.es (2001)

[21] V. Singleton, J. Rossi, Am. J. Enol. Vitic. 16, 144 (1965)

[22] P. Ribéreau-Gayon, E. Stonestreet, Chim. Anal. 48, 188 (1966)

[23] T. Swain, W. Hillis, J. Sci. Food Agric. 10, 63 (1959)

[24] R. Boulton, Am. J. Enol. Vitic. 52, 67 (2001)

[25] J. Valls. Ph.D. thesis. Universitat Rovira i Virgili, (2004)

[26] J.A. Di Rienzo, F. Casanoves, M.G.Balzarini, L. Gonzalez, M. Tablada, C.W Robledo, InfoStat versión 2015. Grupo InfoStat, FCA, Universidad Nacional de Córdoba, Argentina. URL http://www.infostat.com.ar

[27] A. Baiano, G. Terracone, G. Gambacorta, E. La Notte, J. Food Sci. 74, 258 (2009)

[28] P. Ribéreau-Gayon, Y. Glories, A. Maujean, D. Dubordieu, Phenolic Compounds HANDBOOK OF Enology, edited by P. Ribéreau-Gayon and Y. Glories (Wiley, Chichester, 2006), p. 141

[29] S. González-Manzano, M. Dueñas, J.C. RivasGonzalo, M. Escribano-Bailón, C. Santos-Buelga, Food Chem. 114, 649 (2009)

[30] H. Fulcrand, M. Dueñas, E. Salas, V. Cheynier, Am. J. Enol. Vitic. 57, 289 (2006)

[31] E. Salas, M. Dueñas, M. Schwarz, P. Winterhalter, V. Cheynier, H. Fulcrand, J. Agric. Food Chem. 53, 4536 (2005)

[32] M. Dueñas, H. Fulcrand, V. Cheynier, Anal. Chim. Acta 563, 15 (2006)

[33] V. Cheynier, M. Dueñas-Patron, M.J. Souquet, P. Sarni-Manchado, H. Fulcrand, Am. J. Enol. Vitic 57, 298 (2006)

[34] F. He, N. Liang, L. Mu, Q. Pan, J. Wang, J. Reeves, C. Duan, Molec. 17, 1571 (2012)

[35] C.M. Oliveira, A.C. Silva-Ferreira, V. De Freitas, M.S. Silva, Food Res. Int. 44, 1115 (2011) 